\title{
THE IMPACT OF AI ON THE DESIGN OF RECEPTION RoBot: A CASE STUDY
}

\author{
Nguyen Dao Xuan Hai ${ }^{1}$ and Nguyen Truong Thinh ${ }^{2}$ \\ ${ }^{1}$ Faculty of Mechanical Engineering, HCMC University of Technology and \\ Education Ho Chi Minh City, Viet Nam \\ ${ }^{2}$ Department of Mechatronics, HCMC University of Technology and Education \\ Ho Chi Minh City, Viet Nam
}

\begin{abstract}
Service robots have recently drawn a lot of attention from the public. Integrating with the artificial intelligence of computer science, modern service robots have great potential because they are capable of performing many sophisticated human tasks. In this paper, the service robot named "MiABot" as receptionist robot is described, it is a mobile robot with autonomous platform being used with a differential drive and controlled by mini PC. The MiABot could sense its surroundings with the aid of various electronic sensors while mechanical actuators were used to move it around. Robot's behaviour was determined by the program, which was loaded to the microcontrollers and PC with Artificial Intelligence. The experiment results demonstrated the feasibility and advantages of this predictive control on the trajectory tracking of a mobile robot. Service robots are designed to assist humans in reception tasks. Robots will interact closely with a group of people in their daily environment. This means that it is essential to create models for natural and intuitive communication between humans and robots.The theoretical basis of artificial intelligence and its application in the field of natural language processing. Besides, robot software architecture is designed and developed. Robot operation modes and implementation are addressed and discussed, they contain information on algorithm for human robot interacting in natural language, thus a simple approach for generating robot response in arm gesture and emotion. Finally, system evaluation and testing is addressed.
\end{abstract}

\section{KEYWORDS}

AI, Artificial Intelligence, Service Robot, Receptionist Robot, NLP

\section{INTRODUCTION}

The goal of this research was to develop an autonomous robot to assist and give benefit the growing potential user groups at a public reception. The robot used for service purpose was specifically designed with movable, light weight and high stability for long time, supporting of the customers, children or representative of user whom stay in the long distance. As you have known, the service robots, whose purpose is to "educate, entertain, or assist, or protect in the home" (as defined by Robotic Trends magazine) [1], these are appealing significant interest in both the research and commercial communities. Such robots can be particularly useful in addressing the needs of an aging population in industrial societies. To be accepted, these robots need autonomy to interact and communicate with ordinary people in a natural and social way. This defines a new research field coined "Social Robotics". Service robot is among the most promising technology dedicated to supporting the elderly since many countries are now facing aging populations coinciding with a decrease in the amount of the young working population. Service robots assist human beings, generally by performing dirty or tedious work, such as

Dhinaharan Nagamalai et al. (Eds): AIAP, ICCIoT, CNSA, SIGML, IT, ICBB, DMDB - 2020 
household chores. Service robots, in some cases, may replace human caretakers in their ability to care for elderly people. In the paper, the service robot "MiABot" is designed to become a part of the lives of ordinary people. Their tasks may range from entertainment or play to assisting humans with difficult or tedious tasks. In these kinds of applications, the robot will interact closely with a group of humans in their everyday environment. This means that it is essential to create models for natural and intuitive communication between humans and robots. Here we will focus on the use of a mobile service robot, whose main purpose is to help one or more individuals, some of them with special needs, in a home or workplace. The robot will navigate and sense the surrounding in an environment, inhabited by a group of people, and perform interactive tasks, such as communicate to people. It may also keep track of various kinds of information for the users. The robot is partly autonomous, and it carries out its missions in the immediate, shared environment. The robot will navigate and sense the surrounding in an environment, where have a group of people attended and perform interactive tasks, such as communicating with people, to guide a customer to their room from the reception counter. It can also track diversified types of information for individual user when the received theirs room. The robot is an autonomous part, and it performs its tasks in a common, immediate environment. The users, on the other hand, are busy with their ordinary activities while communicating with the robot. The objectives are design and development of autonomous service robot based on a mobile platform to communicate interactive people in open environment as universities, hotels, buildings... and it can move on the smooth floor and avoid the statics and dynamics obstacles as well as finding charge docking based on intelligent algorithms. Several researchers have shown that behaviour-based controllers allow for sophisticated coordination through a utility-centered model of the collective task. Behaviours use this representation to produce actions that consider each robot's impact on the performance of the group as a whole. Autonomous mobile robots are widely used in industry, safe guarding and rescue operations, space exploration, transportation in warehouses, inspection in constrained spaces and services such as office, restaurants, fire and security, people with disabilities, etc. Wheeled Mobile Robots (WMRs) are mechanical devices capable of moving in an environment with a certain degree of autonomy. These robots are increasingly required to navigate and perform purposeful autonomous tasks in more complex domains, where the environment is uncertain and dynamic. A wheeled robot is an autonomous robot and it can autonomously plan and control its own motion in order to accomplish specified tasks. However, these mobile robots are quite restricted in their motion by non-holonomic constraints on their wheel mechanism. The control design of a mobile robot with non-holonomic constraints on the desired path is very difficult in trajectory tracking with determined velocity.

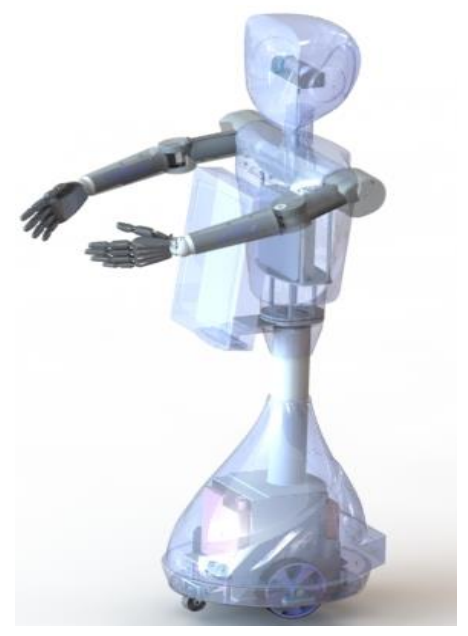

Figure 1. The mechanical structure of MiABot reception robot. 
Nowadays, the researchers have had a significant influence on that field of intelligent service robot, the behaviour-based methods lend itself naturally to robot control problems. The learning by demonstration has also been combined with behaviour-based systems to produce one-shot learning and teaching mechanisms for robot control. The behaviour-based architecture has been used to learn robot navigation strategies; during a learning phase, a trainer selects behaviours for a robot to execute in order to reach a specific goal, and a subsequent offline stage then generates dependency links between the behaviours that it witnessed during the learning phase.

\section{BEHAVIOUR-BASED ARCHITECTURES OF RECEPTION ROBOT}

Designed and developed robot being capable of safely and effectively interacting with humans in real-world is one of the ultimate challenges in now robotics. The robot is utilized a nonholonomic structure, acting as the main driving force for mobile service robot. MiABot's pedestal was a three-wheeled, mobile robot's main wheels powered by 2 servo DC motors based on differential drive mechanism, the other is self-selected wheel, adjusted carefully and automatically by spring force to make sure 3 points in the same plane. The robot consisted of two modules: the body module had a mobile platform and head module had a screen for communicating surrounding people (Figure 1). The two modules were joined by a pan-tilt to form a neck joint for controlling the interaction part with two motors to move the head flexibly based on the customer's height of human face in front of robot. This gave the robot the level of flexibility required for robot when it is in the free movement floors especially in unknown environment. The differential drive mechanism for the wheeled platform provided the ability to negotiate and avoid obstacles. This paper deals with a mobile robot with differential drive which cannot move in the direction lateral to the wheels. The control of this robot is solved by considering its first order kinematics model. The first step to a kinematic model of the robot is to express constraints on the motions of individual wheels. We have designed 2 robotic arms of 5-DoF robot per one to grasp objects or express emotions. There is one motor on the elbow (Pitch), two motors on the shoulder (Roll, Pitch) to lift up a whole arm and by the both sides of the body and two motors on the wrist (Roll, Pitch) to stimulate and imitate exactly the human motions. The MiABot's hand also has five motors not only express the gestures but also grasp the objects or shaking a hand with other human when she received the command, classified in greeting function.

The behaviour-based architecture has been developed for implement and applied in reception robot like as Figure 2. A form of learning by demonstration has used probabilistic methods to select and combine multiple behaviours. The behaviour-based robot has demonstrated various standard capabilities, including obstacle avoidance, navigation, terrain mapping, following, chasing/pursuit, object manipulation, task division and cooperation, and learning maps, navigation and walking.

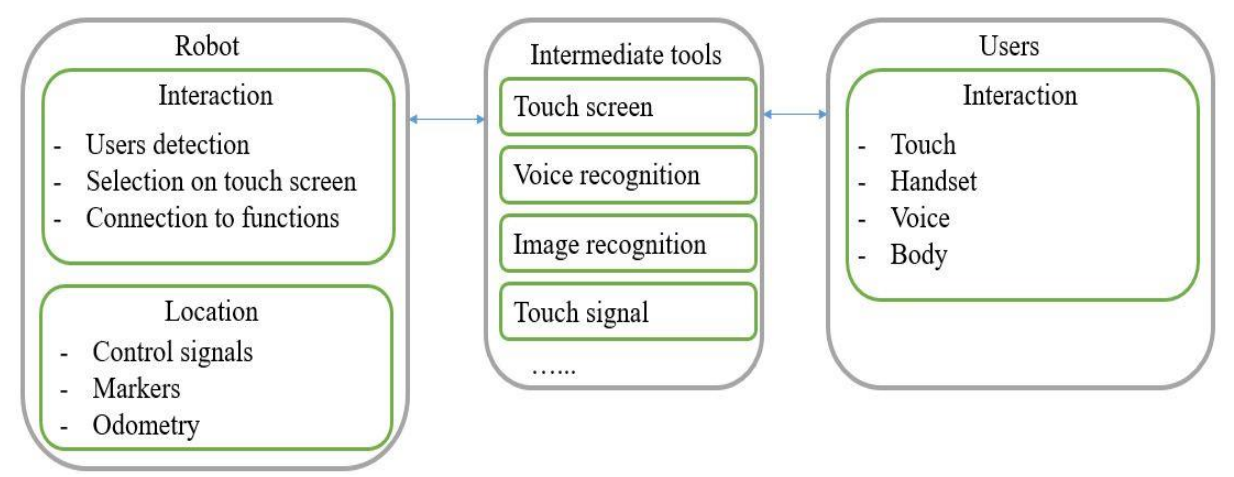

Figure 2. Robot interactive structure. 


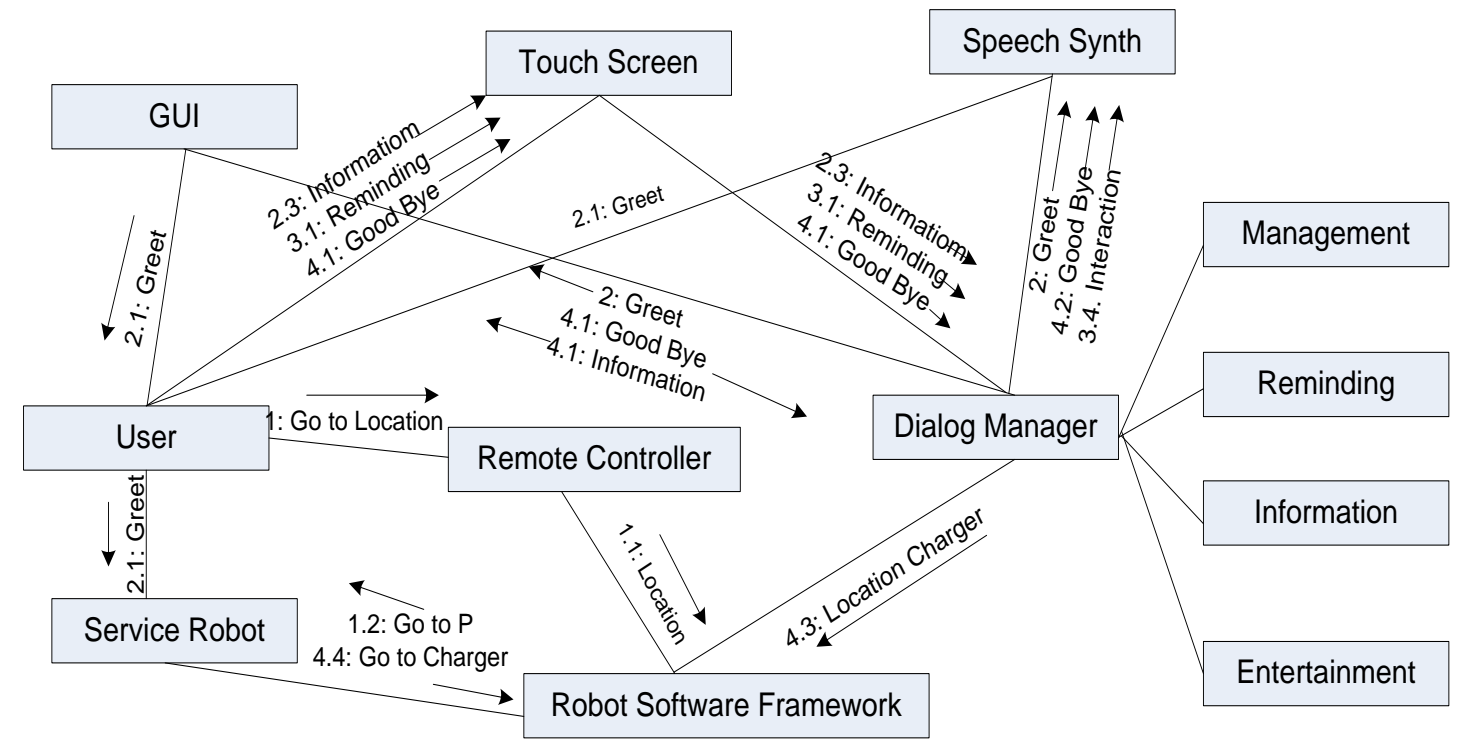

Figure 3. The block diagram of behaviour-based architecture.

Behaviour-based architectures have also been developed for the control and learning an autonomous robot. During a learning phase, the robot observes commands used by a trainer. The behaviour-based methods span mobile robots and being capable of grasping, manipulation, movement, running, and many others. The use of behaviour-based architecture for robot control has evolved from single-system implementations to approaches that combine forms of learning, state estimation, and distributed computation (Figure 3). Behaviours have been combined with a fuzzy inference system for indoor navigation using mobile robots, where a command fusion module acts as an arbiter that combines multiple fuzzy behaviour outputs into a single control signal. This strategy ensures the robot is capable of making inferences in the face of uncertainty.

\section{HUMAN-ROBOT INTERACTION FOR RECEPTION ROBOT}

The facial feature engine is composed of three parts: the face tracking module, the face recognition module and the face detection module, shown the block diagram of face feature engine in Figure 4. In the face tracking module, the face is detected and tracked based on fuzzy controller, assessed by an expert standard and detects each face point in proportion. To embedded the face recognition module, MiABot is allowed to recognize who the person which is trained based on the images using CA-PCA (Principal Component Analysis) [3], CA in this paper which means a model for face processing in advance its adoption by PCs and Cloud Server, model was being trained and developed image recognition algorithm to determine not only forward user face and body detection but also estimates age, gender, expression, and five the other facial emotions. In the face detection module, the final result is the nearest face image from the continuous camera images using $\mathrm{CBCH}$ (Clustering - based Convex Hull) algorithm. MiABot captures surrounding pictures from the camera and sends it to a specific file known as buffer memory, waiting the face detection module to detect the nearest image match with human's face among collected and recognized facial image. Secondly the face image is sent to the face recognition module and face tracking module for processing. The face detection module uses facial feature invariant approach. This algorithm aims to find structural features that exist even when changing the human posture, viewpoint, or even lighting conditions vary was not enough and then use these to locate faces. The algorithm of Haar-like features is applied as the mainly method for face localization alignment to detect, record and training of 
nearly a thousand passenger's faces, these features are judged as fast detection speed, high accuracy and simple calculation.

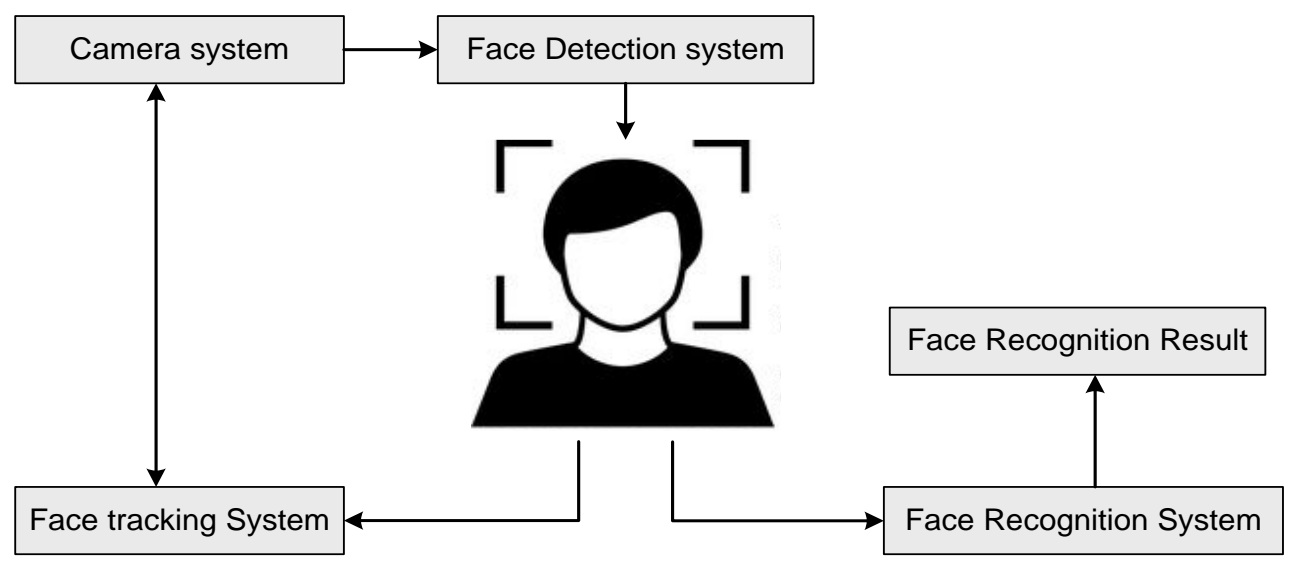

Figure 4. The block diagram of face feature engine.

In evaluating the overall success of a service robot, in this paper MiABot is designed and experimented to assess how stable she is in indoor environments, in the simplest sense, a performance objective must have three important components need to be considered: Autonomous capabilities; meaningful social interaction with the user; being successful in its mission as the receptionist. To solve these issues, components 1 and 3 are being in group and described in term of the localization algorithm implemented for the application. The goal of the proposed localization system is to effect a low-cost accurate navigation system to be applied to a real-world environment. The second component, which is used to appraise the success of a reception robot is its ability to make meaningful interactions with its users.

MiABot can detect users' emotions as well as their age by using facial action analysis through action units have being collected by HD camera to incorporate essential design principles, this is considered as one of the most essential aspects in creating successful assistant robots is the robot-human interaction system (Figure 5). To achieve a successful human-robot interaction, between robots and users, the graphical interface showing a human-like cartoon character has been implemented. The GUI implements as well as the supported functions of the robot, enabling implementation of important design principles, helping to ensure that the robot application can be successfully applied to the real environment. 

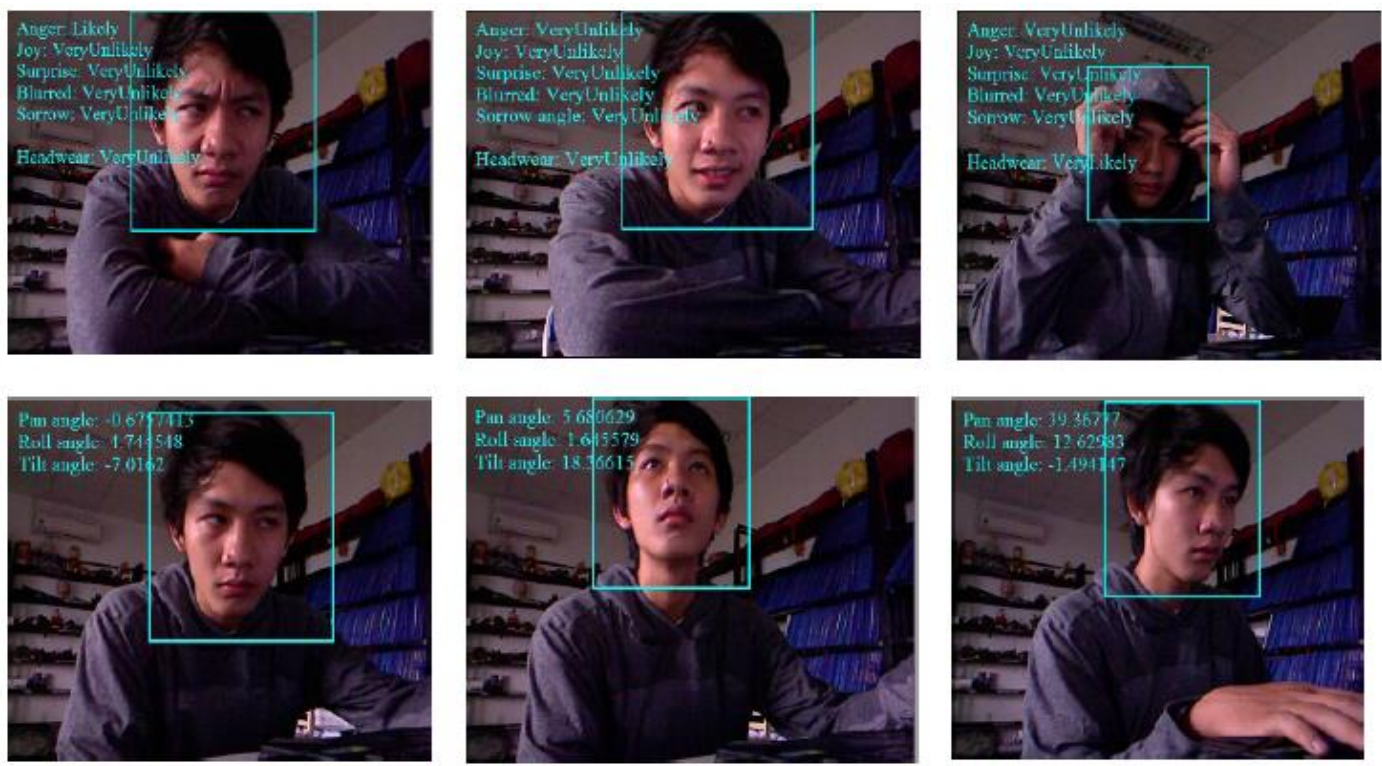

Figure 5. Analysis of facial action via collecting action unit.

\section{Natural Language Processing}

MiABot is also capable of giving direction on hotel lounges, classrooms, and offices within the center for its customers and guests. Besides, the MiABot is also able to remind them of their working schedule and offers user entertainment utilities such as music listening, games based on his/her appearance, emotions and preferences. Furthermore, MiABot is designed with two arms that can handle a wide range of motions and a screen capable of displaying various facial expressions. Robot software needs to be able to coordinate the activities of their actuators so that she response to user command as naturally as possible. MiABot also possesses a touch screen on her chest for displaying information and interacting with her users. The user interface to this touch screen also needs and to design adaptively and responsively for users convenience. As the users could speak different languages, the ability to offer information in many different languages is necessary based on Natural language processing (NLP). NLP is a field which consists of different separate subject such as computer science, artificial intelligence and computational linguistics together with big data algorithm in order to process large natural language corpora (consist for thousands to millions of natural sentence in a specific language) in order to extract useful information from human natural spoken language [2]. The fields range from speech recognition, natural language understand, natural language generator or dialog system (where computer are capable of performing natural conversation between with human). In the field of natural language processing, there are four tasks often considered as the fundamental problems which are: Word tokenization; Part-of-speech tagging; Name entity recognition and Text classification. The capability of understanding Vietnamese and English natural language is essential.

\section{LOCALIZATION AND MOVEMENTS}

In literature, there are three main methods for solving localization. The first is map-based approaches, in which robots use an environment model or a user-created topology model, often called a bed occupancy map. The second approach is to create maps in which robots use its sensors to create geometric models or topologies of the environment and then use these models to navigate. A third approach is to navigate without a map in which robots do not have a clear expression of the environment, but rely on object recognition or object tracking to support 
navigation. For indoor environments, a map-based approach is the most common technique used due to the structural nature of the environment [3]. Robot movements can be grouped into two main categories: normal motion; Avoid obstacles and dynamics. The experiment was carried out on a service robot's mobile platform, which is the ideal test model in Figure 6. They are automatically controlled by an on-board computer, responsible for the smart strategy. The solar sensors connected to the computer are used to achieve position information on static and dynamic obstacles. As a result, this test platform has non-holomonic constraints, storage requirements for the start and end position and direction, and complete monitoring of environmental information. These characteristics are the premise of our control strategy. First, we set the starting position and orientation as well as the starting position and direction. The microprocessor creates a smooth curve from the current position to the end position. Our only concern is whether our robots can track this trajectory well, optimizing the curve is not addressed. The robot has two steering wheels and two wheel balancing wheels. The origin of the Mobile Robot coordinate frame is located at the point between the two rudders. The idea of localization in mapped environment is described through the following method: At each location of the robot orbit, the localization system recognizes the environment by a number of sensor devices. This sensory information is compared to expected values (predicted from an a priori map) and used to correct the location of existing robots, to make sensory data more relevant to data expected data.

\section{EXPERIMENTS AND DISCUSSIONS}

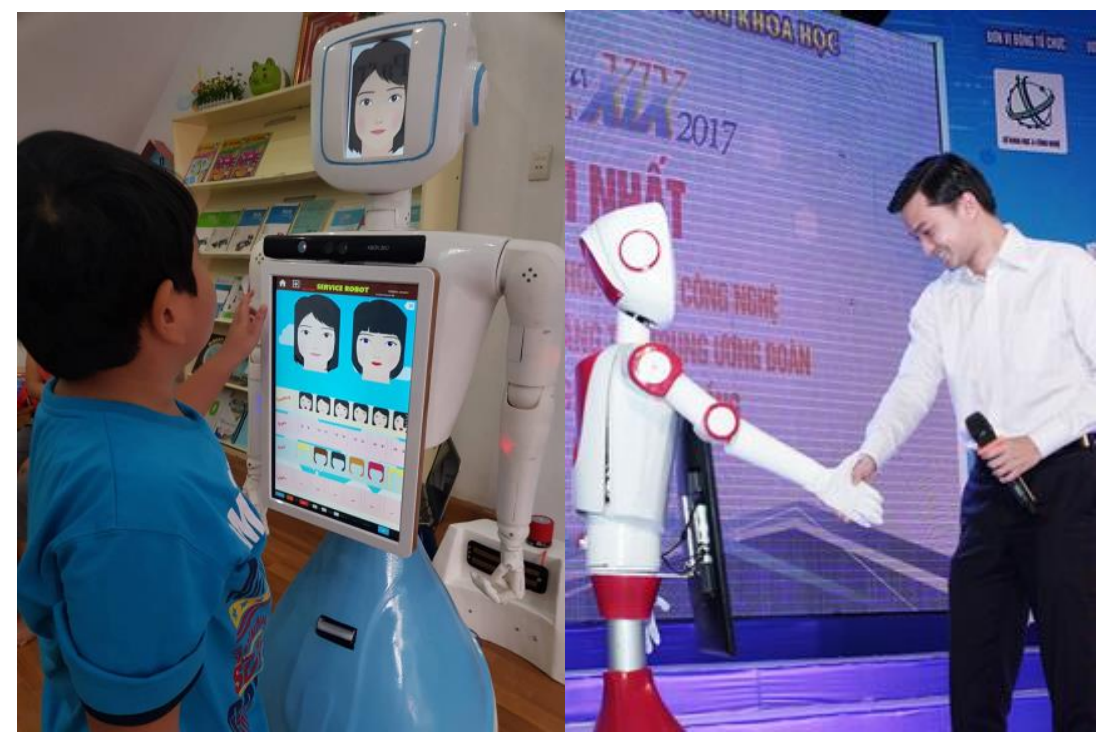

Figure 6. Interaction between the users and MiABot.

The efficient and user-friendly operation interface is one of the most important functions of the service robot as the receptionist (Figure 6). The final goal is to develop an interactive cognition level interface, which utilizes voice and gestures as well as symbolic representation as to the main communication method. In addition, the user may use simple accessories like a handheld controller. The HMI is designed for working with the robot, in some cases cooperating very closely with it. However, the remote control mode is also possible in which the robot is accessible via the Internet. The HMI is designed to be multimedia-based and highly interactive. The model is a simplified 3D description of the environment which include the objects relevant for performing tasks. 


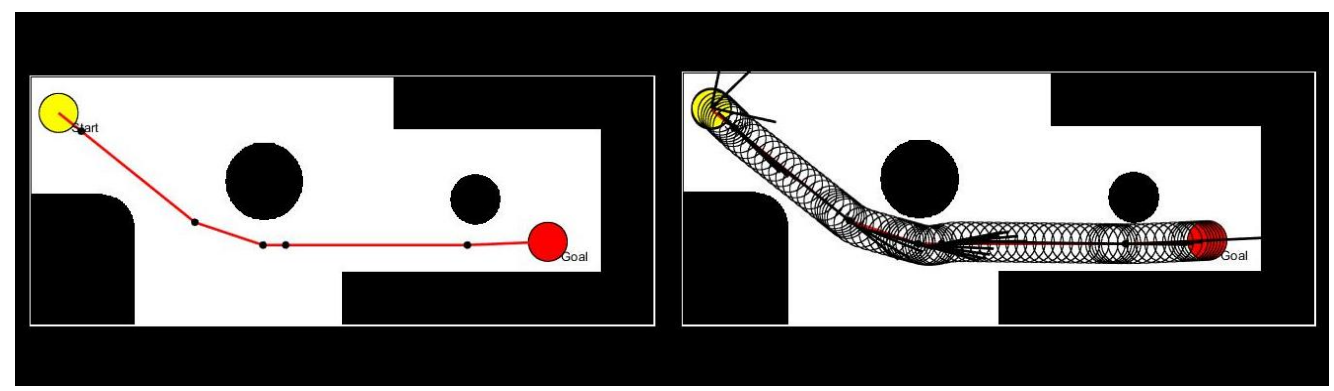

Figure 7. Optimal desired and actual trajectories for robot on the real map.

The main interface of the robot is shown in Fig. 6. Each screen was clearly designed considering for assisting surrounding people. Besides the main interface screen, when the robot communicates, the same sentence is displayed on the screen as subtitles. The speech synthesis module of the robot is based on the Speech Synthesis System tool. It was implemented on the robot in server mode, and the application modules interacted with it using socket communication. In the context of reception mobile robot - MiABot, we have to cope with a number of demanding human-robot interaction tasks which require interaction with the surrounding people based on communication, estimation of age and gender of the interaction person, instructions, consultancy service, and remote surveillance. Besides, image processing helps robot to have a larger vision to the real world. Within image processing, MiABot can recognize people and realize their emotions to make better communication with a human. In this paper, we made two experiments to test the accuracy and convergent efficiency of our method respectively. The basic map of the 3D description is done by the aid of a laser range camera. After geometrical mapping, the relative objects are added to the map by the operator. $\mathrm{MiABot}$ is connected wirelessly both to the user and Internet. Servers on the Internet can serve as knowledge storage shared by many users who are using the same kind of service robot. In the future, the user may find the basic sequence for a new task from the Internet rather than teach it for the robot. The trajectory tracking of MiABot generates the control commands for the robot to follow the previously defined path by taking into account the actual position and orientation, linear and angular velocities, nonholonomic constraint and dynamic constraints imposed by robot. The changes in the terrain topography and texture play a major role in the movements, the MiABot uses ultrasonic sensors to detect and locate the obstacles to choose the best solution to avoid them like as Figure 7. Service robots are designed at a low cost with its dependence on basic sensor fusion including calibration geometry, sonar and monocular vision. The final aspect of development robot testing is robot testing in the target environment with human subjects. Ethical approval was obtained from our lab to conduct an experiment with volunteers. As part of the implementation, 10 volunteers between the ages of 5 and 55 used the function of the Delete MiABot and subsequent interviews were held. Among the volunteers, 100\% thought that the robot was a success and would use it again. $80 \%$ of robots interact without any help. $90 \%$ prefer an interactive system and $40 \%$ think the GUI would be nice. From the interviews, two main errors of the robot system were extracted 1: The interaction distance between the robot and the user is very far (about $150 \mathrm{~cm}$ ). 2: $60 \%$ of users will prefer a taller touch screen, approximately the height of a hand.

\section{Conclusion}

In the paper, we have presented our study where we designed and developed the autonomous mobile robot named MiABot for the intelligent services as the receptionist. The robot was extensively tested by conducting a study in experiments and reality. In this research, the robot interacted with users via synthesized speech and a touch screen, and provided various services and collected data. This paper describes the complete development of a service robot for 
reception primarily through behaviour-based architectures and human-robot interaction systems. The reception robot was applied as a reception and assistant robot and its implementation were discussed and evaluated. The MiABot has described behaviour-based control, a robot control methodology aimed to operate in unconstrained, challenging, and dynamic conditions in the real world. The distributed behaviours are used as the underlying building blocks for these capabilities, allowing behaviour-based systems to take advantage of dynamic interactions with the environment rather than rely solely on explicit reasoning and planning.

\section{ACKNOWLEDGEMENTS}

This research was supported financially by the Ho Chi Minh City University of Technology and Education, Viet Nam.

\section{REFERENCES}

[1] Kwangsu Kim, Hosub Yoon, Robust real-time vision for a personal service robot, Computer Vision and Image Understanding 108 (2007) 196-203.

[2] Luong Huu Thanh Nam, Nguyen Dao Xuan Hai, Truong Cong Toai, and Nguyen Truong Thinh. "Vietnamese Natural Language Processing for Interaction between Human and Robot." In Journal of Technical Education Science, no. 52 (2019): 48-55.

[3] Peter Biber, Tom Duckett, Dynamic Maps for Long-Term Operation of Mobile Service Robots, Wilhelm Schickard Institute for Computer Science, University of Tubingen, Germany. 